Radiologe $2017 \cdot 57: 29-34$

DOI 10.1007/s00117-016-0195-6

Online publiziert: 4. Januar 2017

(c) Der/die Autor(en) 2017. Dieser Artikel ist eine Open-Access-Publikation.

CrossMark

\title{
K. Lampichler
}

Universitätsklinik für Radiologie und Nuklearmedizin, Allgemeines Krankenhaus, Medizinische Universität Wien, Wien, Österreich

\section{Rolle der bildgebenden Verfahren zur Abklärung von Pneumoniekomplikationen}

Ein reduzierter Immunstatus, multiresistente Erreger oder Komorbiditäten können zu teils lebensbedrohlichen Komplikationen wie Lungenempyem, Lungenabszess, Pneumothorax, akutem Lungenversagen („acute respiratory distress syndrome“, ARDS), Multiorganversagen und permanenten Lungenfunktionseinschränkungen führen. Ein rasches Erkennen und Behandeln dieser Komplikationen ist daher essenziell. Neben der physikalischen Untersuchung, klinischen Laborparametern und mikrobiellen Kulturergebnissen ist die radiologische Bildgebung ein essenzieller Pfeiler der Diagnose von Komplikationen.

\section{Lungenempyem}

Die Pneumonie ist eine akute Entzündung des Respirationstrakts und Lungenparenchyms. Initial präsentieren sich Patienten mit typischen Symptomen wie Husten, Dyspnoe, Fieber, pleuritischen Schmerzen und Abgeschlagenheit. Basierend auf unterschiedlichen $\mathrm{Pa}$ tientenkollektiven, Erregerspezies und Behandlungsstrategien werden Pneumonien unterteilt in ambulant erworbene („community-acquired“, CAP), nosokomiale („hospital-acquired“, HAP) und ventilatorassoziierte („ventilator-associated“, VAP) Pneumonien sowie Pneumonien bei immunkompetenten und -supprimierten Patienten. Trotz intensiver und vielfältiger Behandlungsoptionen sind Pneumonien immer noch mit einer deutlichen Morbidität und Mortalität verbunden, im Jahr 2015 waren in Österreich bei $1,1 \%$ aller Todesfälle Pneumonien die Ursache [2].
Pleuraergüsse entstehen durch eine veränderte Permeabilität der Pleura, was zu entweder einem erhöhten hydrostatischen oder erniedrigten onkotischen Druck führt. Dies wird im Rahmen der Pneumonie durch Bakterientoxine und Inflammation ausgelöst. Aufgrund dieser erhöhten Permeabilität können sich auch Erreger aus dem Lungenparenchym in den Pleuraraum ausbreiten und dort eine weitere Infektion - ein Empyem auslösen.

Die Entstehung eines Empyems wird in 3 zeitlich aufeinander folgende Phasen unterteilt. In der exsudativen Phase sammelt sich Flüssigkeit in Form eines parapneumonischen Ergusses mit nur geringer Zellanzahl im Pleuraraum an (• Abb. 1). Als nächstes folgt die fibropurulente Phase, in der sich Fibrin und Granulozyten ablagern und es zu einer Lokulierung des Empyems mit Aus- bildung von Septen kommt. Die letzte Phase führt zu einer Proliferation von Fibroblasten und Narbenbildung, was $\mathrm{zu}$ einer Pleuraschwarte und eventuell zu einer "gefangenen Lunge“ („trapped lung“) führen kann (• Abb. 2).

Das Empyem kann zu weiteren Komplikationen wie der Ausbildung einer bronchopleuralen Fistel oder einem Empyema necessitatis (Ausbreitung der Infektion in die Thoraxwand) führen, weshalb eine antibiotische Behandlung sowie das Legen einer Pleuradrainge oder bei Therapierefraktion auch eine thoraxchirurgische Entfernung erfolgen müssen. Die Mortalitätsrate eines Empyems beträgt immer noch rund $20 \%$.

Pathogene, die häufig - meistens in Kombination mehrerer unterschiedlicher Erregerspezies - zu einem Empyem führen, sind Anaerobier (Bacteroides fragilis, Fusobacterium nucleatum, Prevotella spp.) sowie Streptococcus spp., Staphylococcus aureus (inkl. MRSA), Enterobacteriaceae, Klebsiella pneumoniae und Pseudomonas aeruginosa. Bei immunsupprimierten Patienten kommen zusätzlich noch Mykobakterien, Pilze, Nokardien und Amöben als mögliche Erreger infrage [9, 19].

Die endgültige Diagnose eines Empyems wird durch eine Probenentnahme mit Messung des $\mathrm{pH}$ Werts, klinischchemischen Parametern sowie einer mikrobiologischen und zytologischen Untersuchung gestellt. Durch die septische Entzündung wird Glukose verbraucht $(<40 \mathrm{mg} / \mathrm{dl})$, der $\mathrm{pH}$-Wert sinkt $(<7,2)$ und gleichzeitig steigt die Laktatdehydrogenase (LDH) >1000 IU/L [20, 22]. 

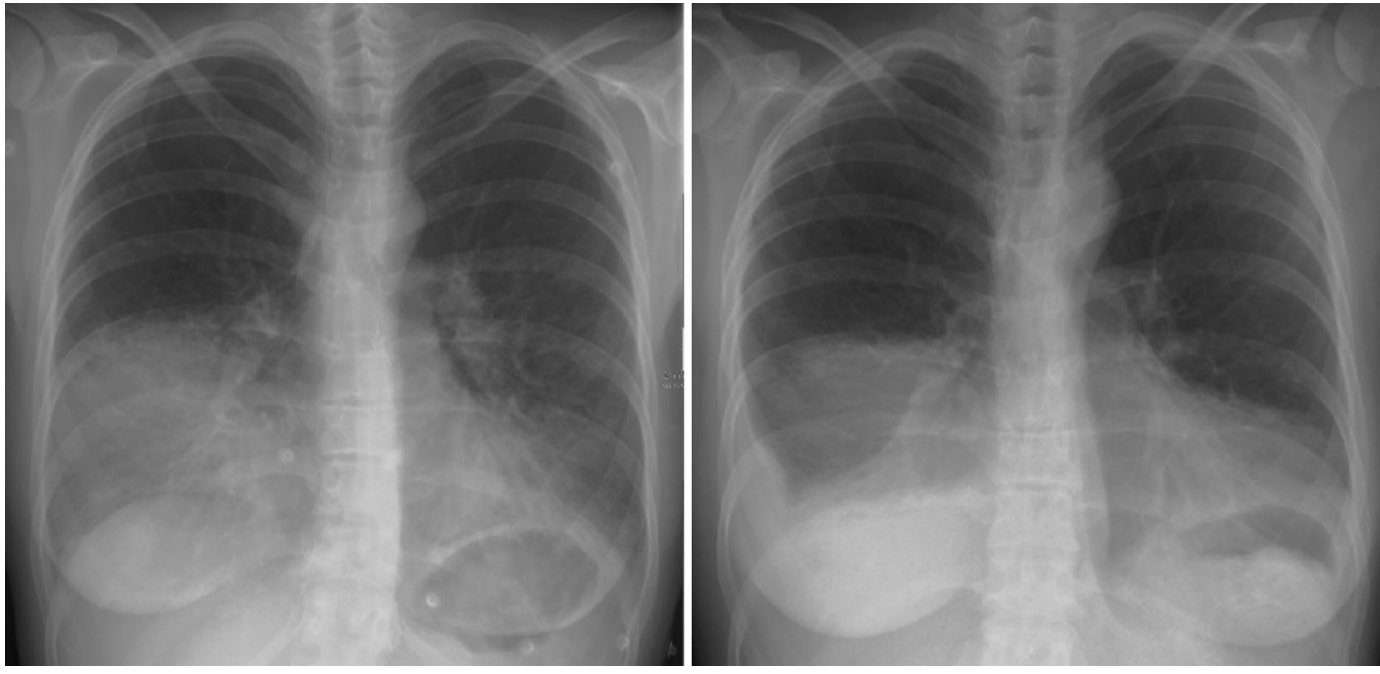

Abb. $1<$ Pleuraempyem im Lungenröntgen; 44-jährige Patientin mit S.-pneumoniae-Pneumonie und Empyembildung innerhalb von 5 Tagen
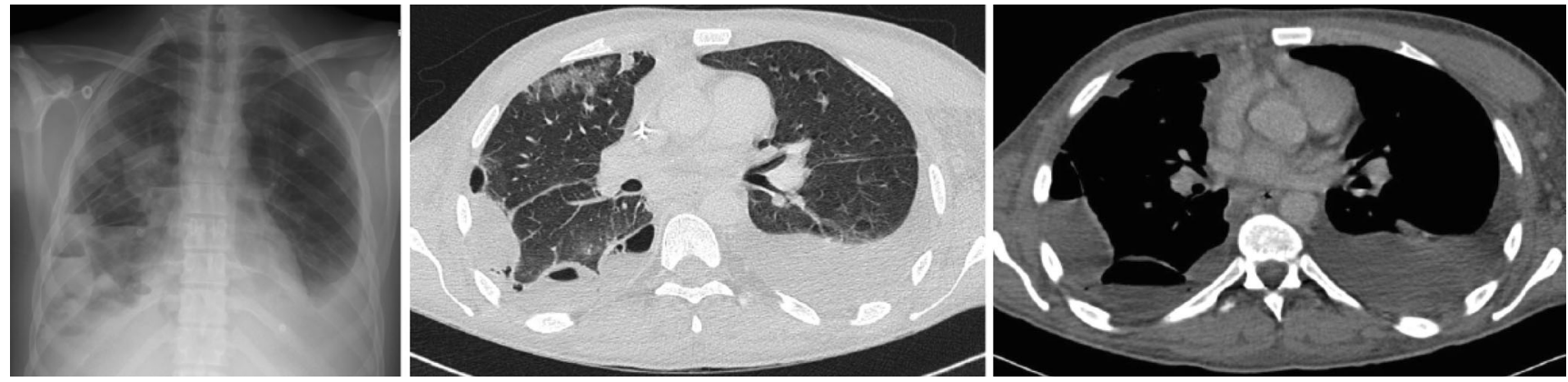

Abb. 2 ॥ Pleuraempyem Stadium II-III mit begleitendem Fluidopneumothorax. 29-jähriger Patient mit S.-aureus-Pneumonie und einem gekämmerten Fluidopneumothorax mit Empyembildung. Aufgrund einer therapierefraktären Sepsis wurde der Patient schließlich dekortiziert und die Diagnose eines organisierenden Empyems mit Pleuraschwarte gestellt

Weder im Lungenröntgen noch in der CT kann die Diagnose eines Empyems letztendlich mit völliger Sicherheit gestellt werden, es gibt allerdings einige deutliche Hinweise auf das Vorhandensein eines Empyems, die an die zuweisende Abteilung kommuniziert werden sollten, um weitere Tests einleiten zu können.

Wichtige Zeichen des Empyems in Lungenröntgen und CT sind in • Tab. 1 aufgelistet. Während die Messung der Dichte eines Pleuraergusses in der CT bei der Diagnose eines Hämatothorax eine wichtige Rolle spielt, ist sie in der Diagnose eines Pleuraempyems nicht hilfreich. Als zusätzliche Untersuchung direkt am Patientenbett eignet sich v. a. der Pleuraultraschall, mit dem sich eine Kämmerung oder weitere Organisation darstellen lässt. Zusätzlich kann auch eine USgezielte Punktion zur Probengewinnung durchgeführt werden $[11,14,16]$.
Zum Ausschluss bzw. Früherkennung eines Pleuraempyems empfehlen die Leitlinien der British Thoracic Society bei allen Patienten mit einem Pleuraerguss von über $5 \mathrm{~cm}$ in der Thoraxseitaufnahme, einen Pleuraultraschall durchzuführen [12].

\section{Pulmonale Abszesse und nekrotisierende Pneumonie}

Durch Pneumonien ausgelöste pulmonale Abszesse sind definiert durch fokale Nekrosen von Lungenparenchym, wobei es zur Abkapselung und Ausbildung einer Kaverne mit einer gut abgrenzbaren, Kontrastmittel aufnehmenden fibrösen Wand kommt. Davon zu trennen ist die nekrotisierende Pneumonie, die ebenfalls durch Nekrose und Liquifizierung von Lungenparenchym innerhalb einer konsolidierten Lunge gekennzeichnet ist, jedoch ohne fibröse Abkapselung. Zusätzlich treten Lungenabszesse meis- tens entweder singulär oder vereinzelt auf, im Rahmen der nekrotisierenden Pneumonie finden sich meist multiple Nekroseareale [5]. Die meisten Lungenabszesse entstehen als Komplikation einer Aspiration. Vor allem Anaerobier, die die physiologische Mund- und Gastrointestinalflora besiedeln, führen zu einer Gewebenekrose mit anschließender Abszedierung.

Häufig abszessbildende Pathogene inkludieren Klebsiella pneumoniae, Escherichia coli, Pseudomonas aeruginosa, Staphylococcus aureus, Streptococcus spp., Fusobacterium, Bacteroides und Prevotella, wobei hier typischerweise mehrere Keime zur Entstehung eines pulmonalen Abszesses beitragen. Zusätzlich können auch v. a. bei immunsupprimierten $\mathrm{Pa}$ tienten Legionella spp., Nokardien, Parasiten und Pilze (Aspergillus, Blastomyces, Histoplasma, Candida spp.) pulmonale Abszesse hervorrufen [3, 25, 27]. 
Klinisch präsentieren sich Patienten mit pulmonalen Abszessen häufig mit seit mehreren Wochen anhaltenden Beschwerden wie Husten, Fieber, pleuritischen Schmerzen, Hämoptysen und purulentem Sputum. Zusätzlich tritt bei etwa einem Drittel ein begleitendes Empyem auf. Im Rahmen der nekrotisierenden Pneumonie kommt es hingegen oft $\mathrm{zu}$ einer raschen Verschlechterung des Gesundheitszustands [17].

Eine Erregerisolation stellt sich oft als schwierig dar, da Blutkulturen bei einer abgekapselten Abszesshöhle nur selten positiv und Sputumproben durch Keime der Mund- und Atemwegsflora kontaminiert sind. Die Behandlung von Lungenabszessen beinhaltet meist eine rein antibiotische Therapie, bei Therapierefraktion können Abszesse auch perkutan drainiert oder chirurgisch saniert werden.

Lungenabszesse sind charakteristischerweise entzündliche Areale, die sich als wandverdickte, runde Kavernen darstellen. Das angrenzende Lungenparenchym weist oft Destruktionen und Konsolidierungen auf. Zusätzlich kann es auch zu Luft-Flüssigkeits-Spiegeln oder Gaseinschlüssen kommen sowie $\mathrm{zu}$ einer Kontrastmittelaufnahme der Kavernenwand.

Im Lungenröntgen ist eine eindeutige Kavernierung manchmal schwierig $\mathrm{zu}$ erkennen und kann mit einem Empyem verwechselt werden. Die CT weist eine deutlich höhere Sensitivität auch für die Detektion kleinerer Kavernen auf (• Abb. 3; [15]). CT-morphologische Differenzierungen zwischen Empyem und Lungenabszess sind in - Tab. 2 zusammengefasst.

Die frühzeitige Erkennung von Lungenabszessen mithilfe von Lungenröntgen oder CT kann Therapieentscheidungen und -verlauf maßgebend beeinflussen. Neben den primären Lungenabszessen aufgrund von Pneumonien können Lungenabszesse auch sekundär auftreten, meist in Form septischer Emboli - hier zeigen sich meist mehrere Kavernen, wohingegen bei primären Abszessen nur einzelne wenige Kavernen angrenzend oder direkt in dem von der Pneumonie betroffenen Lungenparenchym auftreten.

Radiologe 2017·57:29-34 DOI 10.1007/s00117-016-0195-6

(c) Der/die Autor(en) 2017. Dieser Artikel ist eine Open-Access-Publikation.

\section{K. Lampichler}

\section{Rolle der bildgebenden Verfahren zur Abklärung von Pneumoniekomplikationen}

\section{Zusammenfassung}

Klinisches Problem. Pneumonien sind

trotz antimikrobieller Therapien und multidisziplinärer Behandlungsstrategien unverändert mit einer hohen Morbiditäts- und Mortalitätsrate verbunden. Eine Infektion des Respirationstrakts kann zu Komplikationen wie Lungenempyem, -abszess und akutem Lungenversagen führen. Zusätzlich kommt es neben den intrapulmonalen Komplikationen häufig auch zur Beeinträchtigung anderer Organe. Kardiovaskuläre Grunderkrankungen wie Herzinsuffizienz, Arteriosklerose oder Rhythmusstörungen können sich im Rahmen einer Pneumonie entweder neu manifestieren oder infektbedingt verschlimmert werden. Eine frühzeitige Diagnose und Therapie dieser teils lebensbedrohlichen Komplikationen sind entscheidend und beeinflussen den Therapieerfolg maßgeblich.

Radiologische Standardverfahren. Zu den wichtigsten bildgebenden Verfahren zählen das Lungenröntgen und die Computertomographie des Thorax. Eine eindeutige Charakterisierung der Pathologie ist sowohl im Lungenröntgen als auch in der CT manchmal schwierig oder gar nicht möglich, trotzdem ergeben sich wichtige Hinweise auf intra- und extrapulmonale Komplikationen einer Pneumonie, die mithilfe der Radiologie frühzeitig erkannt werden können. Als zusätzliche portable Untersuchungsmodalität direkt am Patienten eignet sich der Pleuraultraschall zur weiteren Diagnosesicherung und Interventionshilfestellung.

Empfehlung für die Praxis. Das Lungenröntgen ist sowohl als unkomplizierte initiale Bildgebung als auch zur Kontrolle des Therapieverlaufs wichtig. Bei Verdacht auf Komplikationen, Fortschreiten der Erkrankung oder Diskrepanz zur klinischen Symptomatik sollte eine weiterführende CT des Thorax durchgeführt werden.

Schlüsselwörter Lungenröntgen · Computertomographie . Akutes Lungenversagen - Empyem . Lungenabszess

\section{Role of imaging procedures in clarification of complications of pneumonia}

\section{Abstract}

Clinical issue. Despite a considerable number of antimicrobial agents and interdisciplinary treatment options, lower respiratory tract infections are still associated with high morbidity and mortality rates. Infections of the respiratory tract can lead to severe complications, such as empyema, lung abscesses and acute respiratory distress syndrome (ARDS). Besides intrapulmonary complications pneumonia can also impair other organs due to a systemic inflammatory response. Underlying cardiovascular diseases, such as chronic heart failure, arteriosclerosis and dysrhythmia can either deteriorate due to infections or be newly manifested as a result of pneumonia. Early diagnosis and therapy of these sometimes life-threatening complications are crucial and can have a severe impact on disease outcome. Standard radiological methods. The most important imaging techniques include chest
X-ray and computed tomography (CT) of the chest. Although a definite diagnosis is sometimes difficult or even impossible to establish using chest X-ray or CT, there are several findings indicative of intrapulmonary or extrapulmonary complications of pneumonia. Another useful and portable tool is thoracic ultrasound directly on the patient, which can be used to further define the underlying disease or as guidance during procedures.

Practical recommendations. The chest X-ray is useful in initial diagnosis and follow-up. If complications or disease progression are suspected or the clinical course differs from the X-ray interpretation, a subsequent $\mathrm{CT}$ of the chest should be performed.

Keywords

Chest $\mathrm{X}$ ray $\cdot$ Computed tomography $\cdot$ Human ARDS $\cdot$ Empyema $\cdot$ Lung abscess 
Tab. 1 Morphologische Hinweise auf ein Pleuraempyem in Lungenröntgen und CT

\begin{tabular}{lll}
$\begin{array}{l}\text { Lungen- } \\
\text { röntgen } \\
\text { CT }\end{array}$ & - & $\begin{array}{l}\text { Linsenförmige pleurale Verschattung } \\
\text { Stumpfer Winkel zur Thoraxwand }\end{array}$ \\
Stadium I & $\begin{array}{l}\text { Pleuraerguss ohne verdickte Pleura } \\
\text { Angrenzendes Lungenparenchym komprimiert }\end{array}$ \\
\hline Stadium III & $\begin{array}{l}\text { Kämmerung } \\
\text { Verbreiterung der Pleura (Pleura gleichmäßig verdickt und Kontrastmit- } \\
\text { tel aufnehmend) } \\
\text { "Split pleura sign“ (Flüssigkeit zwischen viszeraler und parietaler Pleura) } \\
\text { Eventuell Gaseinschlüsse }\end{array}$ \\
\hline $\begin{array}{l}\text { Zunehmend verdickte und Kontrastmittel aufnehmende Pleura } \\
\text { Verbreiterung des epipleuralen Fetts }\end{array}$
\end{tabular}

\section{Tab. 3 Akutes Lungenversagen im Lungenröntgen und CT}

Lungen- Verschattungen meist bilateral und symmetrisch

röntgen Unscharf begrenzte Hili

Im Vergleich zum kardiopulmonalen Ödem gibt es zeitliche Veränderungen und

keine Kardiomegalie

Keine Kerley-B-Linien

Periphere alveolären Verschattungen

CT Anterior-posteriorer Gradient der Konsolidierungen (dorsal am stärksten) Ventrale Lunge entweder normal oder überbläht (bei mechanischer Ventilation) Traktionsbronchiektasien

Neu aufgetretene Konsolidierungen in ventralen Abschnitten oft Zeichen eines zusätzlichen Infekts

\section{Diffuser Alveolarschaden und akutes Lungenversagen}

Ein akutes Lungenversagen liegt bei $\mathrm{Pa}$ tienten mit bilateralen pulmonalen Infiltraten und einer arteriellen Hypoxie ohne Hinweis auf andere hämodynamische oder kardiologische Ursachen vor. Die Pneumonie gilt als Hauptursache des akuten Lungenversagens, oft auch aggraviert durch Aspiration, Sepsis oder Trauma [29].

Um für den Gasaustausch ein optimal trockenes Klima zu schaffen, ist das normale Epithel der Alveolen auch für kleinste lösliche Stoffe impermeabel. Bei Patienten mit akutem Lungenversagen kommt es durch entzündliche Schädigung des Alveolarepithels zum Eindringen von Flüssigkeit in den Alveolarraum und somit zu einem pulmonalen Ödem (diffuser Alveolarschaden). Dies kann durch zusätzlichen biophysikalischen Stress im Rahmen einer künstlichen Beatmung weiter verstärkt werden [21].

Es wird angenommen, dass das akute Lungenversagen einen stadienhaften Verlauf aufweist, beginnend mit dem diffusen Alveolarschaden mit intraalveolärem Ödem und Blutung sowie Atelektasen. Ab etwa 72 h kommt es zu weiteren pathologischen Veränderungen durch Infiltration von Monozyten, was zu einer Hyperplasie der Epithelzellen und interstitieller Fibrose führen kann. Zusätzlich gibt es allerdings auch Formen des akuten Lungenversagens ohne Nachweis eines akuten Alveolarschadens. Hier wird aus histopathologischer Sicht primär die Pneumonie selbst als Ursache des akuten Lungenversagens genannt $[18,28]$. Der stadienhafte Verlauf auf zellulärer Ebene kann auch zur Interpretation von Lungenröntgen und CT verwendet werden.

Obwohl es keine spezifischen bildmorphologischen Zeichen eines akuten Lungenversagens gibt, sind radiologische Verfahren essenziell in Diagnosestellung, Evaluierung des Therapieansprechens und zur Darstellung weiterer Komplikationen [26].

Da Patienten mit akutem Lungenversagen oft intensivmedizinisch betreut werden müssen, ist das Lungenröntgen als portable Modalität ein wertvoller Test zur Überwachung des Krankheitsverlaufs. Obwohl das akute Lungenversagen keine rein radiologische Diagnose ist, gibt es einzelne Lungenveränderungen, die eher das Vorhandensein eines akuten Lungenversagens als an-
Tab. 2 Unterscheidung zwischen Lun-

genabszess und Pleuraempyem

Lungen- Rundlich

abszess Ungleichmäßig verdickte Wand Lungenparenchym zerstört Spitzer Winkel zur Thoraxwand

Pleura- Linsenförmig

empyem Gleichmäßig verdickte Wand Angrenzende Lunge komprimiert Stumpfer Winkel zur Thoraxwand

Tab. 4 Kardiale Komplikationen nach

Häufigkeit

Komplikationen

Häufigkeit

(\%)

Verschlechterung der Herzin- 14

suffizienz

Arrhythmien

5

Akutes Koronarsyndrom 5

(ACS)

Herzstillstand 3

dere mögliche Differenzialdiagnosen (Pneumonie, Lungenblutung, Stauung) darstellen (• Tab. 3). Zusätzlich können auch neu eingebrachte Zugänge einfach kontrolliert werden.

In der CT zeigt sich ein anteriorposteriorer Gradient mit dorsal immer weiter zunehmenden Konsolidierungen (• Abb.4). Milchglasverschattungen sind ein uncharakteristisches Zeichen für eine reduzierte Pneumatisierung des Lungenabschnitts. Im Rahmen des akuten Lungenversagens stellt Milchglas v. a. Abschnitte mit flüssigkeits- und proteingefüllten Alveolen und Interstitium dar. Zeichen des akuten Lungenversagens in der CT sind in - Tab. 1 zusammengefasst. $\mathrm{Zu}$ den Spätfolgen des „acute respiratory distress syndrome" (ARDS) und der Pneumonie zählen Fibrose, Lungenzysten und Bullae $[10,13]$.

Zusammenfassend spielen sowohl Lungenröntgen als auch CT eine essenzielle Rolle bei der Erkennung, Überwachung und Nachsorge von Patienten mit ARDS.

\section{Kardiale Komplikationen}

Pneumonien können einerseits bereits bestehende kardiale Erkrankungen ver- 

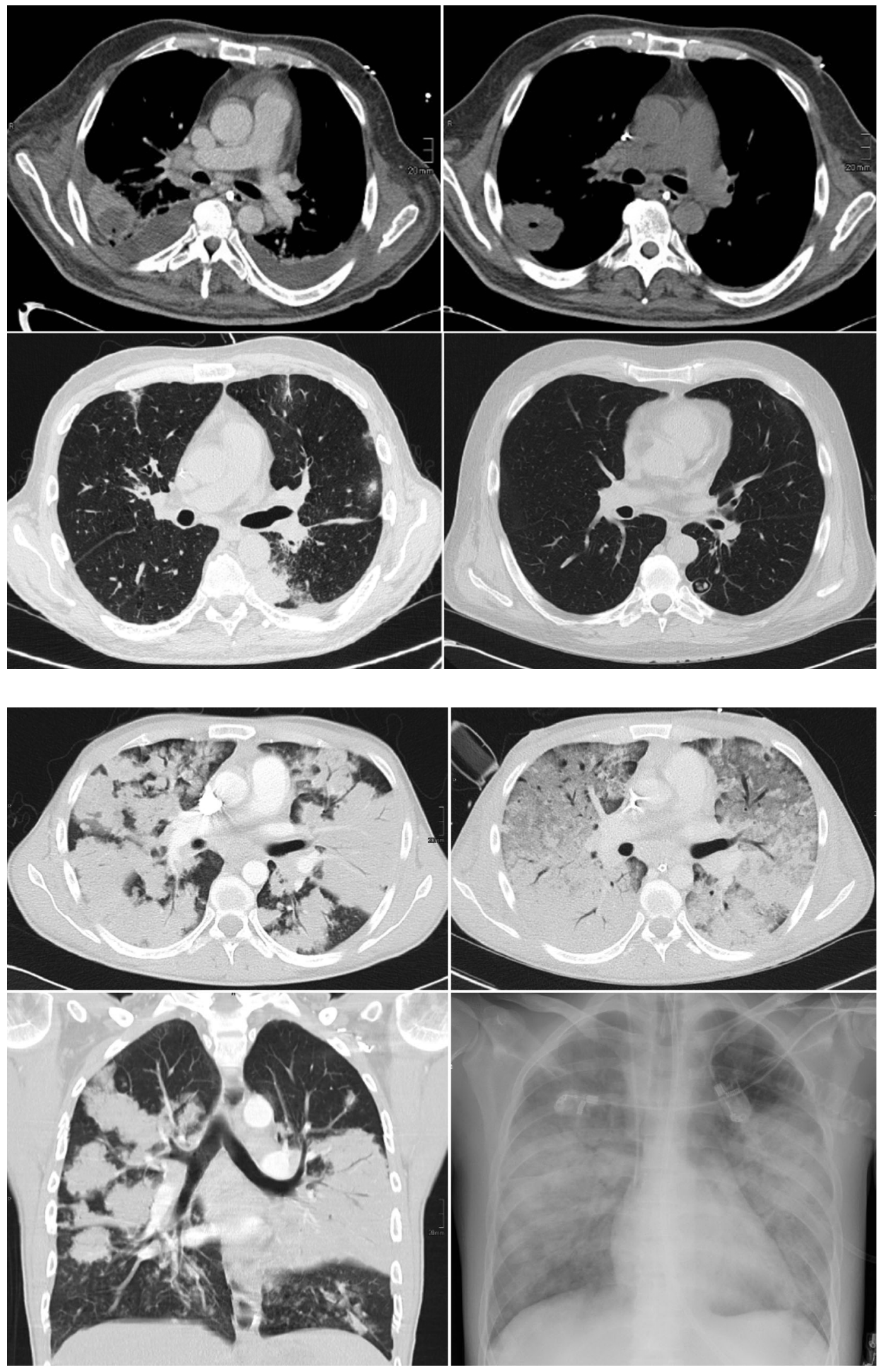

Abb. $3<$ Lungenabszess und Aspergillom. Obere Reihe 53-jähriger Patient nach Knochenmarktransplantation. K.-pneumoniae-Pneumonie mit Bildung eines einzelnen Lungenabszesses (10-Tages-Verlauf). Untere Reihe 40-jähriger Patient mit einer kavernierenden Asperigillus-Pneumonie im Rahmen einer invasiven Aspergillose. Die größte Kaverne bildete zusätzlich die Grundlage für ein späteres Aspergillom
Abb. $4 \triangleleft$ Akutes Lungenversagen, 30-jähriger Patient mit S.-pneumoniaePneumonie (linke Spalte) und Entwicklung eines akuten Lungenversagens innerhalb von $48 \mathrm{~h}$ (rechte Spalte) 
stärken, andererseits allerdings auch zusätzliche neu auftretende kardiale Komplikationen hervorrufen. Bei einer Pneumonie kommt es nicht nur zu einer lokalen Entzündung, sondern ebenfalls zu einer signifikanten systemischen Immunantwort, die neben den Lungen auch weitere Organe wie das Herz betreffen können.

Aufgrund des erhöhten Sauerstoffbedarfs, aber erniedrigten Sauerstofftransports in das Myokard kommt es zu einer Ischämie und veränderten Myokardfunktion mit verringerter Kontraktilität und Konduktion. Folgen davon können Herzversagen, Arrhythmien (v. a. Vorhofflimmern) und ein akutes Koronarsyndrom sowie ein akuter Myokardinfarkt sein. Bereits vorhandene kardiologische Grunderkrankungen können verstärkt werden. Ein zusätzlicher Risikofaktor ist eine bestehende Koronarsklerose, wobei es hier durch die Pneumonie zu einer erhöhten Gefahr einer Plaqueruptur oder Thrombusformation kommt [1, 6, 7].

Durch eine entzündlich getriggerte Thrombozytose entsteht ein Zustand der Hyperkoagobilität. Dies ist ein weiterer Faktor, der zu einer erhöhten Inzidenz akuter kardialer Ereignisse führen kann [23].

Als Risikofaktoren gelten hohes Alter, vorbekannte kardiovaskuläre Vorerkrankungen und ausgedehnte Pneumonien. Neunzig Prozent der kardialen Komplikationen treten in den ersten 7 Tagen auf [4, 24].

Einige wichtige kardiale Komplikationen zeigt - Tab. 4; [8].

\section{Fazit für die Praxis}

- Pneumonien sind trotz fortschrittlicher Therapieoptionen immer noch mit einer deutlichen Morbidität und Mortalität verbunden.

- Im Rahmen der Infektion kann es zu unterschiedlichen intra- und extrapulmonalen Komplikationen kommen. Hierzu zählen Lungenempyem, Lungenabszess, akutes Lungenversagen und kardiovaskuläre Ereignisse.

- Die Radiologie spielt eine zentrale Rolle in der Früherkennung und The- rapieplanung von Komplikationen der Pneumonie.

\section{Korrespondenzadresse}

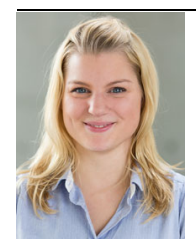

Mag. Dr. K. Lampichler

Universitätsklinik

für Radiologie und

Nuklearmedizin, Allgemeines

Krankenhaus, Medizinische

Universität Wien

Währinger Gürtel 18-20,

1090 Wien, Österreich

katharina.lampichler@

meduniwien.ac.at

Open access funding provided by Medical University of Vienna.

\section{Einhaltung ethischer Richtlinien}

Interessenkonflikt. K. Lampichler gibt an, dass kein Interessenkonflikt besteht.

Dieser Beitrag beinhaltet keine von den Autoren durchgeführten Studien an Menschen oder Tieren.

Open Access Dieser Artikel wird unter der Creative Commons Namensnennung 4.0 International Lizenz (http://creativecommons.org/licenses/by/4.0/deed. de) veröffentlicht, welche die Nutzung, Vervielfältigung, Bearbeitung, Verbreitung und Wiedergabe in jeglichem Medium und Format erlaubt, sofern Sie den/die ursprünglichen Autor(en) und die Quelle ordnungsgemäßnennen, einen Link zur Creative Commons Lizenz beifügen und angeben, ob Änderungen vorgenommen wurden.

\section{Literatur}

1. Aliberti S, Ramirez JA (2014) Cardiac diseases complicating community-acquired pneumonia. Curr Opin Infect Dis 27:295-301

2. Anonymous (2015) Statistik Austria. Todesursachen im Überblick. Anaerobe

3. Bartlett JG (2012) Anaerobic bacterial infection of the lung. Anaerobe 18:235-239

4. Bordon J, Wiemken T, Peyrani P et al (2010) Decrease in long-term survival for hospitalized patients with community-acquired pneumonia. Chest 138:279-283

5. Chatha N, Fortin D, Bosma KJ (2014) Management of necrotizing pneumonia and pulmonary gangrene: a case series and review of the literature. Can Respir J21:239-245

8. Corrales-Medina VF, Suh KN, Rose G et al (2011) Cardiac complications in patients with community-acquired pneumonia: a systematic review and meta-analysis of observational studies. PLOSMed 8:e1001048

7. Corrales-Medina VF, Musher DM, Wells GA et al (2012) Cardiac complications in patients with community-acquired pneumonia: incidence, timing, risk factors, and association with shortterm mortality. Circulation 125:773-781

6. Corrales-Medina VF, Musher DM, Shachkina S et a (2013) Acute pneumonia and the cardiovascular system. Lancet 381:496-505
9. DesaiH, Agrawal A (2012)Pulmonaryemergencies: pneumonia, acute respiratory distress syndrome, lung abscess, and empyema. Med Clin North Am 96:1127-1148

10. Desai SR (2002) Acute respiratory distress syndrome: imaging of the injured lung. Clin Radiol 57:8-17

11. Evans AL, Gleeson FV (2004) Radiology in pleural disease: state of the art. Respirology 9:300-312

12. Hooper C, Lee YC, Maskell N et al (2010) Investigation of a unilateral pleural effusion in adults: British Thoracic Society Pleural Disease Guideline 2010. Thorax 65(Suppl 2):4-17

13. Joynt GM, Antonio GE, Lam P et al (2004) Latestage adult respiratory distress syndrome caused by severe acute respiratory syndrome: abnormal findingsatthin-section CT. Radiology 230:339-346

14. Kearney SE, Davies CW, Davies RJ et al (2000) Computed tomography and ultrasound in parapneumonic effusions and empyema. Clin Radiol 55:542-547

15. Kelogrigoris M, Tsagouli P, Stathopoulos K et al (2011) CT-guided percutaneous drainage of lung abscesses: review of 40 cases. JBR-BTR 94:191-195

16. Kraus GJ (2007) The split pleura sign. Radiology 243:297-298

17. Kuhajda I,Zarogoulidis K, Tsirgogianni Ketal (2015) Lung abscess-etiology, diagnostic and treatment options. Ann Transl Med 3:183

18. Lorente JA, Cardinal-Fernandez P, Munoz D et al (2015) Acute respiratory distress syndrome in patients with and without diffuse alveolar damage: an autopsy study. Intensive Care Med 41:1921-1930

19. Mansharamani NG, Koziel H (2003) Chronic lung sepsis: lung abscess, bronchiectasis, and empyema. Curr Opin Pulm Med 9:181-185

20. Maskell N, British Thoracic Society Pleural Disease Guideline (2010) British Thoracic Society Pleural Disease Guidelines - 2010 update. Thorax 65:667-669

21. Matthay MA, Ware LB, Zimmerman GA (2012) The acute respiratory distress syndrome. J Clin Invest 122:2731-2740

22. Mccauley L, Dean N (2015) Pneumonia and empyema: causal, casual or unknown. J Thorac Dis 7:992-998

23. Mirsaeidi M, Peyrani $P$, Aliberti S et al (2010) Thrombocytopenia and thrombocytosis at time of hospitalization predict mortality in patients with community-acquired pneumonia. Chest 137:416-420

24. Mortensen EM, Kapoor WN, Chang CC et al (2003) Assessment of mortality after long-term follow-up of patients with community-acquired pneumonia. Clin Infect Dis 37:1617-1624

25. Pande A, Nasir S, Rueda AM et al (2012) The incidence of necrotizing changes in adults with pneumococcal pneumonia. Clin Infect Dis 54:10-16

26. Pesenti A, Musch G, Lichtenstein D et al (2016) Imaging in acute respiratory distress syndrome. Intensive Care Med 42:686-698

27. Takayanagi N, Kagiyama N, Ishiguro T et al (2010) Etiology and outcome of community-acquired lung abscess. Respiration 80:98-105

28. Thille AW, Esteban A, Fernandez-Segoviano $P$ et al (2013) Comparison of the Berlin definition for acute respiratory distress syndrome with autopsy. Am J Respir Crit Care Med 187:761-767

29. Ware LB, Matthay MA (2000) The acute respiratory distress syndrome. N Engl J Med 342:1334-1349 